\title{
Modeling Phase Separation in Nonstoichiometric Silica
}

\author{
V. M. Burlakov, ${ }^{1, *}$ G. A. D. Briggs, ${ }^{1}$ A. P. Sutton, ${ }^{1, \dagger}$ Angelo Bongiorno, ${ }^{2}$ and Alfredo Pasquarello ${ }^{2}$ \\ ${ }^{1}$ Department of Materials, University of Oxford, Parks Road, Oxford OXI 3PH, United Kingdom \\ ${ }^{2}$ Institute de Théorie des Phénoménes Physiques (ITP), Ecole Polytechnique Fédérale de Lausanne (EPDL), CH-1015 Switzerland \\ and Institut de Recherche Numérique en Physique des Matériaux (IRRMA), CH-1015 Lausanne, Switzerland
}

(Received 7 October 2003; published 20 September 2004)

\begin{abstract}
We have modeled the decomposition of nonstoichiometric amorphous $\mathrm{SiO}_{x}$ upon annealing into silicon and stoichiometric silica, using a new method based on mapping Metropolis Monte Carlo simulations onto rate equations. The concentrations of all oxidation states of silicon are derived as a function of time and found to attain steady-state values at long times dependent on temperature $T$ and oxygen content $x$. The degree of phase separation and the sizes of Si particles are predicted as a function of $T$ and $x$, enabling greater control over the size of silicon quantum dots in silica matrices.
\end{abstract}

DOI: 10.1103/PhysRevLett.93.135501

PACS numbers: 61.43.Bn, 68.35.-p

Nonstoichiometric amorphous silica $\left(\mathrm{SiO}_{x}\right)$ has attracted interest in a wide range of fields within physical science (see, for example, [1-6]). It is also widely used in industry for protective layers, optical coatings, insulating layers, and gas-barrier layers [7-11]. Some experimental studies [1] suggest the bulk $\mathrm{SiO}_{x}$ with $x \sim 1$ consists of a mixture of pure $\mathrm{SiO}_{2}$ and $\mathrm{Si}$ phases, separated by layers comprising $\mathrm{Si}$ atoms in intermediate oxidation states. Other experiments [2-4] in which amorphous $\mathrm{SiO}_{2} / \mathrm{SiO} / \mathrm{SiO}_{2}$ superlattices were annealed yielded a homogeneous array of $\mathrm{Si}$ quantum dots within the $\mathrm{Si}-\mathrm{O}$ layers, the size of the dots depending on the layer thickness and annealing conditions. These results suggest that $\mathrm{SiO}_{x}$ tends to separate into pure $\mathrm{Si}$ and $\mathrm{SiO}_{2}$. In this Letter, we present a new and remarkably simple method to model the decomposition of $\mathrm{SiO}_{x}$ into pure $\mathrm{Si}$ and $\mathrm{SiO}_{2}$ as a function of time, temperature, and composition. We find that the degree of phase separation reaches a constant value at long annealing times, and we predict steady-state sizes of $\mathrm{Si}$ particles in $\mathrm{SiO}_{2}$ matrices and of $\mathrm{SiO}_{2}$ particles in Si matrices as a function of $T$ and $x$ (Fig. 3).

Assuming that (a) each oxygen atom is always bonded to two silicon atoms, and (b) each silicon atom has four nearest neighbors, then the numbers of $\mathrm{Si}-\mathrm{O}$ and $\mathrm{Si}-\mathrm{Si}$ bonds in $\mathrm{SiO}_{x}$ are conserved throughout separation. Therefore, there must be an additional contribution to the energy of the system beyond a sum of Si-O and $\mathrm{Si}$ $\mathrm{Si}$ bond energies to drive separation. The origin of this additional term is nonpairwise and it is known as the suboxide penalty energy (a Si atom with 1,2, or 3 oxygen neighbors is called a silicon suboxide). Suboxide penalty energies were obtained $a b$ initio either from periodic suboxide models [12] or from suitable $\mathrm{Si}-\mathrm{O}$ clusters [13].

The Metropolis Monte Carlo (MC) technique with moves involving the exchange of pairs of nearest neighbors of bonded $\mathrm{Si}$ atoms [14] is widely used for simulating amorphous $\mathrm{Si}$ and $\mathrm{SiO}_{2}$ structures and interfaces between them [15]. In this Letter, we apply this approach to nonstoichiometric silica. To obtain an acceptance ratio of at least $1 \%$ we find that the simulation temperature has to be very high, and at some compositions it is almost certainly above the real melting point. The simulation of phase separation is very slow even at such high temperatures. We present a new approach to model the phase separation by mapping the Metropolis Monte Carlo procedure onto rate equations for average concentrations of all possible oxidation states (OS) of $\mathrm{Si}$ in $\mathrm{SiO}_{2}$. Our approach enables us to model phase separation at realistic temperatures over much longer times than can be achieved in Monte Carlo simulations for the same CPU time.

Phase separation in nonstoichiometric silica is effected by diffusion of oxygen. Consider a $\mathrm{Si}$ atom with $1 \leq k \leq 3$ oxygen nearest neighbors, so that of its four nearest neighbors, at least one is an oxygen atom and at least one is a silicon atom. The value of $k$ is defined as the $\mathrm{OS}$ of the $\mathrm{Si}$ atom, and $\mathrm{Si}$ atoms with $k$ oxygen neighbors are denoted by $\operatorname{Si}(k)$. Consider a bond between a $\mathrm{Si}(k)$ atom and one of its $k$ neighboring oxygen atoms. Let the OS of the other $\mathrm{Si}$ atom to which the oxygen is bonded be $i$, where $1 \leq i \leq 4$. Let one of the $\mathrm{Si}-\mathrm{Si}$ bonds to the same $\operatorname{Si}(k)$ atom be to a $\operatorname{Si}(j)$ atom, where $0 \leq j \leq 3$. The fundamental diffusional step involves translation of the oxygen atom from the bond between $\mathrm{Si}(k)$ and $\operatorname{Si}(i)$ and insertion into the adjacent bond between $\operatorname{Si}(k)$ and $\operatorname{Si}(j)$. After such a step $k$ does not change, but $i \rightarrow i-1$ and $j \rightarrow j+1$. Assuming a random distribution of all OS, the probabilities of choosing a neighboring pair, $\operatorname{Si}(k)$, and $\operatorname{Si}(l)$, which have $\left(P_{k l}^{\text {ox }}\right)$ or have not $\left(P_{k l}^{\text {nox }}\right)$, a common oxygen atom between them are

$$
\begin{aligned}
P_{k l}^{\mathrm{ox}}(t) & =\frac{k l}{4} \frac{n_{k}(t) n_{l}(t)}{Z^{\mathrm{ox}}(t)}, \\
P_{k l}^{\mathrm{nox}}(t) & =\frac{(4-k)(4-l)}{4} \frac{n_{k}(t) n_{l}(t)}{Z^{\max }(t)},
\end{aligned}
$$

where $n_{k}(t)$ and $n_{l}(t)$ are the concentrations of $\operatorname{Si}(k)$ and $\mathrm{Si}(l)$ atoms at time $t$. Since the overall probability of finding $\operatorname{Si}(k)$ is $\sum_{l}\left[P_{k l}^{\mathrm{ox}}(t)+P_{k l}^{\mathrm{nox}}(t)\right]=n_{k}(t)$, the normal- 
ization factors are given by

$$
Z^{\mathrm{ox}}(t)=\sum_{l} \ln _{l}(t), \quad Z^{\mathrm{nox}}(t)=\sum_{l}(4-l) n_{l}(t) .
$$

The probability $\tilde{P}_{i j}$ that an oxygen atom bonded to a $\mathrm{Si}(i)$ atom neighbors a $\mathrm{Si}-\mathrm{Si}$ bond to a $\mathrm{Si}(j)$ atom can then be defined as

$$
\tilde{P}_{i j}(t)=\frac{n_{i}(t) n_{j}(t) i(4-j)}{12 Z_{\mathrm{ox}}(t) Z_{\mathrm{nox}}(t)} \sum_{k} n_{k}(t) k(4-k) .
$$

We follow Metropolis MC and assume that the probability of an oxygen jump depends on the energy difference between the initial and the final states of the system. Oxygen diffusion is much slower than the associated relaxation processes; therefore, we assume that the system is always elastically relaxed. The probability of an oxygen jump is governed only by the suboxide penalty energies $\Delta_{i}$ in the initial and final states. The energies $\Delta_{i}$ for $\operatorname{Si}(i)$ are from Ref. [13]: $\Delta_{1}=0.5 \mathrm{eV}, \Delta_{2}=0.51 \mathrm{eV}$, $\Delta_{3}=0.22 \mathrm{eV}$, and $\Delta_{0}=\Delta_{4}=0$. The probability $W_{i, j}(t)$ that at time $t$ an oxygen atom bonded to a $\mathrm{Si}(i)$ atom jumps into a neighboring $\mathrm{Si}-\mathrm{Si}$ bond to a $\mathrm{Si}(j)$ atom is thus

$$
W_{i, j}(t)=\frac{\tilde{P}_{i j}(t)}{Z} \min \left\{1, \exp \left(-\frac{E_{\text {final }}-E_{\text {init }}}{k T}\right)\right\},
$$

where $Z$ is a normalization constant with which we determine the time scale, $k$ is the Boltzmann constant, and $T$ is the system temperature. The factor min $\left\{1, \exp \left[-\left(E_{\text {final }}-E_{\text {init }}\right) / k T\right]\right\}$ in Eq. (4) is equal to unity if the energy of the final state $E_{\text {final }}=\Delta_{i-1}+\Delta_{j+1}$ is lower than that of the initial state $E_{\text {init }}=\Delta_{i}+\Delta_{j}$, otherwise it is equal to the Boltzmann factor.

The evolution of the concentration of oxidation state $n_{i}(t)$ is determined by a set of coupled nonlinear rate equations:

$$
\begin{aligned}
\frac{d n_{i}(t)}{d t}= & \sum_{j=0}^{3} W_{i, j}(t)+\sum_{j=0}^{3} W_{i+1, j}(t)+\sum_{j=1}^{4} W_{j, i-1}(t) \\
& -\sum_{j=1}^{4} W_{j, i}(t) .
\end{aligned}
$$

Equations (1)-(5) are solved numerically using some initial concentrations of the oxidation states at temperature $T$. To validate our rate-equation (RE) model, we compared its solutions with results obtained from MC simulations for the same annealing temperature. First we generated a number of amorphous $\mathrm{SiO}_{x}$ structures by simulated deposition using our recently developed method [16]. At this stage, the suboxide penalty energies were set to zero. The structures were then annealed for about $10^{6} \mathrm{MC}$ steps taking into account suboxide penalty energies. The annealing temperature was optimized for each particular structure to have a reasonable acceptance rate of MC moves. During annealing, moves included both the motion of dangling bonds [16] and the switching of pairs of bonds [14], the combination enabling more complete relaxation of the elastic energy.

Using the initial OS concentrations obtained immediately after deposition of amorphous $\mathrm{SiO}_{x}$, we calculated their evolution during annealing using our RE approach for comparison with our MC simulations. Figure $1 \mathrm{com}-$ pares the results obtained by both methods for $\mathrm{SiO}_{1.7}$ and $\mathrm{SiO}_{0.33}$. It is seen that the RE model reproduces the results of the MC simulations well. In addition, we found that for a given composition, the final concentrations of OS obtained within the RE model do not depend on their initial values, indicating that a true steady state is reached. This steady-state solution describes an equilibrium configuration of the system. It is not the ground-state configuration of the system, which would display some degree of crystallization. Such a configuration is inaccessible in our model because we neglect the strain energy.

Our rate-equation approach is more than a million times faster than the Metropolis Monte Carlo simulations. As seen in Fig. 1, we are able to extend the simulation time of the phase separation process by several orders of magnitude using the rate equations.

Figure 2 shows the $x$ dependence of the steady-state OS concentrations in annealed $\mathrm{SiO}_{x}$. We see that at all $x$, the
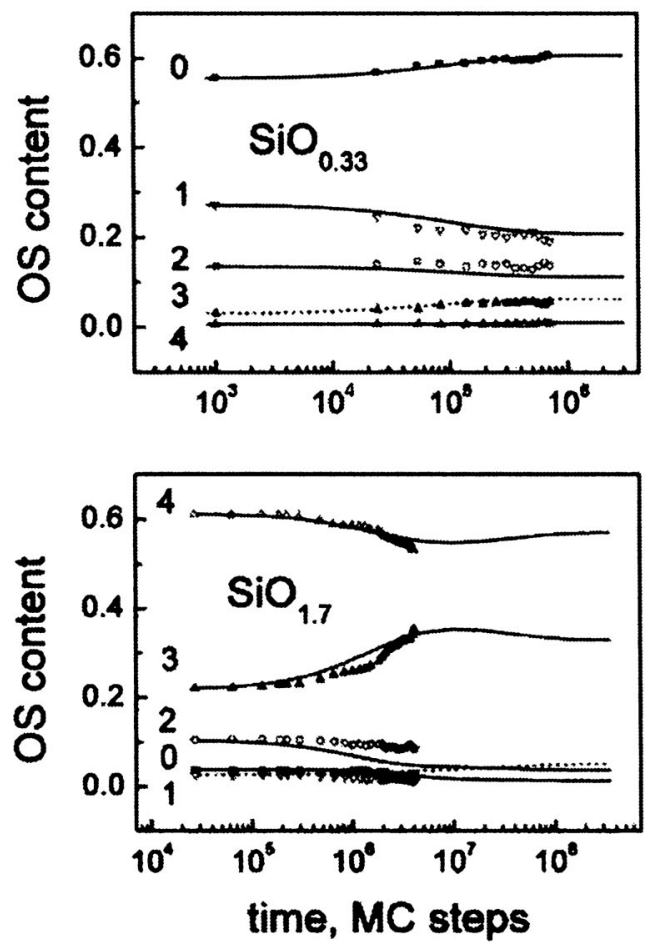

FIG. 1. Time evolution of OS concentrations in amorphous $\mathrm{SiO}_{0.33}$ and $\mathrm{SiO}_{1.7}$. Symbols: $\mathrm{MC}$ results for $k T=$ $0.35 \mathrm{eV}\left(\mathrm{SiO}_{0.33}\right)$ and $k T=0.15 \mathrm{eV}\left(\mathrm{SiO}_{1.7}\right)$. Curves: RE results for the same temperatures. The time scale in the RE model is adjusted through the parameter $Z$ in Eq. (4) to give best fit to $\mathrm{MC}$ results. Curves are labeled according to OS. 


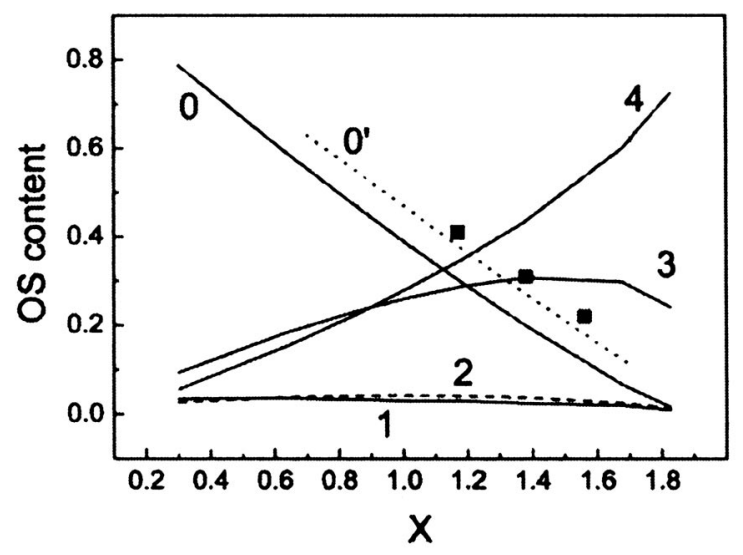

FIG. 2. Steady-state OS concentrations as a function of $x$. Curves labeled $0,1,2,3$, and 4 correspond to $\operatorname{Si}(i)$, and were obtained within the RE model [using Eqs. (6) and (7)] at $T=$ $1250{ }^{\circ} \mathrm{C}$. Symbols represent experimental data for the content of $\mathrm{Si}(0)$ in the bulk $\mathrm{SiO}_{x}$ annealed at $T=1250{ }^{\circ} \mathrm{C}$ [18]. The curve $0^{\prime}$ shows $\mathrm{RE}$ results for $\operatorname{Si}(0)$ at $T \approx 600{ }^{\circ} \mathrm{C}$.

system comprises primarily $\mathrm{Si}(0), \mathrm{Si}(4)$, and suboxide $\mathrm{Si}(3)$, indicating phase separation into $\mathrm{Si}$ and $\mathrm{SiO}_{2}$ with interfaces containing $\mathrm{Si}(3)$. The dominance of $\mathrm{Si}(3)$ at these interfaces is consistent with experimental observations reported in [17]. We found that the concentrations of $\mathrm{Si}(1)$ and $\mathrm{Si}(2)$ start to rise only at temperatures above $\sim 1200{ }^{\circ} \mathrm{C}$. In Fig. 2, we also show experimental values for $\mathrm{Si}(0)$ content obtained after annealing $\mathrm{SiO}_{x}$ at $T=$ $1250{ }^{\circ} \mathrm{C}$ for about $1 \mathrm{~h} \mathrm{[18]}$. The experimental values are slightly higher than those calculated for $T=1250{ }^{\circ} \mathrm{C}$ within the RE model.

We may estimate the size of pure Si clusters by calculating an average surface-to-volume ratio for the clusters, corresponding to the ratio of the number of $\mathrm{Si}$ atoms with fewer than four $\mathrm{Si}(0)$ neighbors to the number of $\mathrm{Si}(0)$ atoms within the clusters. Since we have assumed a random distribution of OS, the probability $P_{S}$ for a $\mathrm{Si}$ atom to be on the cluster surface, i.e., to have $k=1$ or 2 neighboring suboxides $\operatorname{Si}(i)(i=1,2,3)$, and the probability $P_{\mathrm{V}}$ for a $\mathrm{Si}$ atom to be anywhere in the cluster are

$$
P_{S} \simeq \sum_{k=1}^{2} \frac{4 !}{k !(4-k) !} P_{0}^{k} P_{1}^{4-k}, \quad P_{V}=P_{S}+P_{0}^{4}
$$

where $P_{0}=4 n_{0}, P_{1}=\sum_{i=1}^{3}(4-i) n_{i}$, and $n_{i}$ is the steady-state concentration of the $i$ th OS. To avoid overestimation of $\mathrm{Si}$ clusters surface area, we do not count $\mathrm{Si}(0)$ atoms having three neighboring suboxides as belonging to Si clusters. As we will be interested in the ratio of $P_{S} / P_{V}$, the probabilities $P_{S}$ and $P_{V}$ do not have to be normalized. Assuming that all clusters are identical spheres of diameter $D_{\mathrm{cl}}$, we obtain $D_{\mathrm{cl}}=2 R$ from the equation

$$
P_{S} / P_{V}=\left[R^{3}-(R-d)^{3}\right] / R^{3} .
$$

The value of the thickness $d$ of a monatomic layer on the cluster surface can be estimated as $d=\rho^{-1 / 3} \simeq 0.27 \mathrm{~nm}$, where the density of amorphous $\mathrm{Si}$ is taken to be $\rho \simeq$ 0.05 atoms $/{ }^{3}$. Substituting the steady-state concentrations of OS at $T=1250{ }^{\circ} \mathrm{C}$ into Eqs. (6) and (7), we obtain the diameter of Si clusters as a function of $x$, shown by the broken line in Fig. 3. The symbols in Fig. 3 show experimentally determined average diameters of Si nanocrystals at $T=1250{ }^{\circ} \mathrm{C}$ and $1100{ }^{\circ} \mathrm{C}$.

It is seen in Fig. 3 that the experimental values of nanocrystal sizes are about 2 times higher than those estimated using Eqs. (6) and (7) for a temperature $T=$ $1250{ }^{\circ} \mathrm{C}$. This discrepancy indicates that our assumption of a random distribution of OS [implicit in the use of $W_{i, j}(t)$ as a parameter independent of cluster size] breaks down. One possible reason is Ostwald ripening [19], which would decrease the surface-to volume ratio, as compared with the prediction of Eq. (6). However, we note that the experimental observations of steady-state cluster sizes after sufficiently long anneals indicate that the ripening process is eventually suppressed, presumably by the development of elastic accommodation stresses, which are directly proportional to the cluster volume [20].

Despite the breakdown of our assumption of a random distribution of OS, we find that the increase in the average cluster size can still be described by the RE model by reducing the temperature at which phase separation is assumed to occur. This is seen in Fig. 3 where the solid line shows the prediction for an assumed growth temperature of $\sim 600{ }^{\circ} \mathrm{C}$. A similar improvement between the prediction of the RE model and experiment for the overall content of $\mathrm{Si}$ phase is obtained also with a growth temperature of $\sim 600{ }^{\circ} \mathrm{C}$ (curve $0^{\prime}$ in Fig. 2). These observations indicate that the influence of nonrandomness

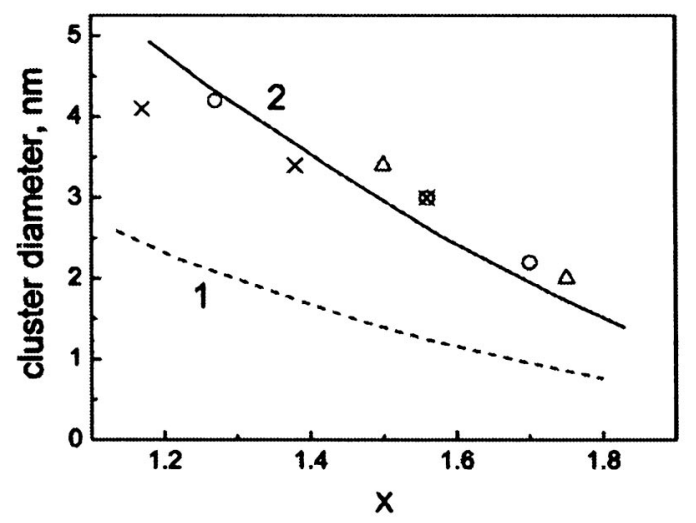

FIG. 3. Lines: average Si cluster diameter as a function of $x$ at (1) $T=1250{ }^{\circ} \mathrm{C}$ and $(2) \sim 600{ }^{\circ} \mathrm{C}$, as calculated using Eqs. (6) and (7). Symbols are experimental values of the average size of Si nanocrystals: circles [21] and crosses [18] are for bulk $\mathrm{SiO}_{x}$ annealed at $T=1250{ }^{\circ} \mathrm{C}$, triangles are for $\mathrm{SiO}_{2} / \mathrm{SiO} / \mathrm{SiO}_{2}$ superlattice annealed at $T=1100{ }^{\circ} \mathrm{C}$ [3]. 
of the distributions of OS in real systems may be reproduced in the RE model by assuming a lower temperature of phase separation, or, according to Eq. (4), correspondingly higher suboxide penalty energies.

In conclusion, we have derived a set of rate equations based on the Metropolis Monte Carlo procedure, and shown that $\mathrm{SiO}_{x}$ tends to separate into $\mathrm{Si}$ and $\mathrm{SiO}_{2}$ clusters at all temperatures of experimental relevance. Our rate equations enabled phase separation throughout more of the temperature-oxygen content space to be explored than can be modeled with Monte Carlo simulations. The solution of the rate-equation yielded steady-state concentrations of various OS of $\mathrm{Si}$, the degree of phase separation, and the expected sizes of Si particles. This work provides a means of predicting the sizes of $\mathrm{Si}$ quantum dots embedded in $\mathrm{SiO}_{2}$, formed by annealing $\mathrm{SiO}_{x}$ at various values of $x$ and temperature, as seen experimentally in Refs. [2-4]. Similar analysis can be performed also for $\mathrm{SiO}_{2}$ particles in $\mathrm{Si}$ matrix.

V. M. B., G. A. D. B., and A. P. S. acknowledge the support of the Toppan Printing Co., Ltd. A. B. and A.P. acknowledge the support of the Swiss Science Foundation (Grant No. 620-57850.99) and the Swiss Center for Scientific Computing (SCSC).

*On leave from the Institute for Spectroscopy, Russian Academy of Sciences, Troitsk, Moscow region, 142090, Russia

${ }^{\dagger}$ Also Helsinki University of Technology, Laboratory of Computational Engineering, PO Box 9203, FIN-02015 HUT, Finland.

[1] T. Hohl, P. A. Wieder, T. E. van Aken, G. Weirich, M. Denninger, S. Vidal, C. Oswald, J. Deneke, H. Mayer, and J. Fuess, J. Non-Cryst. Solids 320, 255 (2003).

[2] M. Zacharias, J. Heitmann, and U. Goesele, MRS Bulletin Vol. 26 No. 12, edited by Luis M. Liz-Marzan and David Norris (p. 975, Dec. 2000).
[3] M. Zacharias, J. Heitmann, R. Scholz, U. Kahler, M. Schmidt, and J. Biasing, Appl. Phys. Lett. 80, 661 (2002).

[4] L. X. Yi, J. Heitmann, R. Scholz, and M. Zacharias, Appl. Phys. Lett. 81, 4248 (2002).

[5] K. Sato, T. Izumi, M. Iwase, Y. Show, H. Morisaki, T. Yaguchi, and T. Kamino, Appl. Surf. Sci. 216, 376 (2003).

[6] S. Wada, Y. Murata, A. T. Tokunaga, and J. Watanabe, Astron. Astrophys. 406, 783 (2003).

[7] G. Hassand and C. D. Salzberg, J. Opt. Soc. Am. 44, 181 (1954).

[8] M. J. O'Leary and J. H. Thomas III, J. Vac. Sci. Technol. A 5, 106 (1987).

[9] J. U. Schmidt and B. Schmidt, Mater. Sci. Eng., B 101, 28 (2003).

[10] C. A. Wang, R. K. Huang, D. A. Shiau, M. K. Connors, P. G. Murphy, P.W. O’Brien, A.C. Anderson, D. M. DePoy, G. Nichols, and M. N. Palmisiano, Appl. Phys. Lett. 83, 1286 (2003).

[11] M.S. Hedenqvist and K.S. Johansson, Surf. Coat. Technol. 172, 7 (2003).

[12] D. R. Hamann, Phys. Rev. B 61, 9899 (2000).

[13] A. Bongiorno and A. Pasquarello, Phys. Rev. B 62, R16326 (2000).

[14] F. Wooten, K. Winer, and D. Weaire, Phys. Rev. Lett. 54, 1392 (1985).

[15] Y. Tu and J. Tersoff, Phys. Rev. Lett. 84, 4393 (2000).

[16] V. M. Burlakov, G. A. D. Briggs, A. P. Sutton, and Y. Tsukahara, Phys. Rev. Lett. 86, 3052 (2001).

[17] F. Rochet, Ch. Poncey, G. Dufour, H. Roulet, C. Guillot, and F. Sirotti, J. Non-Cryst. Solids 216, 148 (1997); A. Barranco, F. Yubero, J. P. Espinos, J. P. Caballero, A. R. Gonzalez-Elipe, and J. A. Mejias, Vacuum 67, 491 (2002).

[18] L. Dal Negro, M. Cazzanelli, N. Daldosso, Z. Gaburro, L. Pavesi, F. Priolo, D. Pacifici, G. Franzò, and F. Iacona, Physica E (Amsterdam) 16, 297 (2003).

[19] A. P. Sutton and R.W. Balluffi, Interfaces in Crystalline Materials (Oxford University Press, New York, 1995), p. 628-633.

[20] J. D. Eshelby, Proc. R. Soc. London A 241, 376 (1957).

[21] F. Iacona, G. Franzò, and C. Spinella, J. Appl. Phys. 87, 1295 (2000). 